\title{
Bilateral Tactile Input Patterns Decoded at Comparable Levels But Different Time Scales in Neocortical Neurons
}

\author{
Clara Genna, ${ }^{1}$ Calogero M. Oddo, ${ }^{1}$ Alberto Mazzoni, ${ }^{1}$ Anders Wahlbom, ${ }^{2}$ Silvestro Micera, ${ }^{1,3}$ and Henrik Jörntell ${ }^{2}$ \\ ${ }^{1}$ The BioRobotics Institute, Scuola Superiore Sant'Anna, 56025 Pisa, Italy, ${ }^{2}$ Neural Basis of Sensorimotor Control, Department of Experimental Medical \\ Science, Lund University, SE-221 84 Lund, Sweden, and ${ }^{3}$ Bertarelli Foundation Chair in Translational NeuroEngineering, Center for Neuroprosthetics and \\ Institute of Bioengineering, School of Engineering, École Polytechnique Federale de Lausanne, 1015 Lausanne, Switzerland
}

\begin{abstract}
The presence of contralateral tactile input can profoundly affect ipsilateral tactile perception, and unilateral stroke in somatosensory areas can result in bilateral tactile deficits, suggesting that bilateral tactile integration is an important part of brain function. Although previous studies have shown that bilateral tactile inputs exist and that there are neural interactions between inputs from the two sides, no previous study explored to what extent the local neuronal circuitry processing contains detailed information about the nature of the tactile input from the two sides. To address this question, we used a recently introduced approach to deliver a set of electrical, reproducible, tactile afferent, spatiotemporal activation patterns, which permits a high-resolution analysis of the neuronal decoding capacity, to the skin of the second forepaw digits of the anesthetized male rat. Surprisingly, we found that individual neurons of the primary somatosensory can decode contralateral and ipsilateral input patterns to comparable extents. Although the contralateral input was stronger and more rapidly decoded, given sufficient poststimulus processing time, ipsilateral decoding levels essentially caught up to contralateral levels. Moreover, there was a weak but significant correlation for neurons with high decoding performance for contralateral tactile input to also perform well on decoding ipsilateral input. Our findings shed new light on the brain mechanisms underlying bimanual haptic integration.
\end{abstract}

Key words: bilateral; haptic; information processing; neocortex; neuron; neurophysiology

Significance Statement

Here we demonstrate that the spiking activity of single neocortical neurons in the somatosensory cortex of the rat can be used to decode patterned tactile stimuli delivered to the distal ventral skin of the second forepaw digits on both sides of the body. Even though comparable levels of decoding of the tactile input were achieved faster for contralateral input, given sufficient integration time each neuron was found to decode ipsilateral input with a comparable level of accuracy. Given that the neocortical neurons could decode ipsilateral inputs with such small differences between the patterns suggests that $S 1$ cortex has access to very precise information about ipsilateral events. The findings shed new light on possible network mechanisms underlying bimanual haptic processing.

\section{Introduction}

The organization of the somatosensory cortex has generally been described as a somatotopic representation of the contralateral

\footnotetext{
Received Oct. 6, 2017; revised Feb. 12, 2018; accepted March 6, 2018.

Author contributions: C.G., C.M.O., A.W., S.M., and H.J. designed research; C.G. performed research; C.G. and A.M. contributed unpublished reagents/analytic tools; C.G., C.M.O., A.M., and H.J. analyzed data; C.G., C.O., A.M., A.W., S.M., and H.J. wrote the paper.

This work was supported by the Ministry of Education, Universities and Research of the Italian Republic; and the Swedish Research Council, via the Italy-Sweden bilateral Research Project J52l15000030005 SensBrain (Brain Network Mechanisms for Integration of Natural Tactile Input Patterns); by EU Grant FET 611687 NEBIAS Project (NEUrocontrolled BIdirectional Artificial upper limb and hand prosthesiS); by EU Grant FP7-NMP 228844 NANOBIOTOUCH project (Nanoresolved Multi-Scan Investigations of Human Tactile Sensations and Tissue Engineered Nanobiosensors); and by National Project Grant B81J12002680008 PRIN/HandBot (Biomechatronic hand prostheses endowed with bio-inspired tactile perception, bidirectional neural interfaces, and distributed sensori-motor control), Hjärnfonden, and the Swedish Research Council (Project Grant K2014-63X-14780-12-3).
}

(CL) body surface (Nieuwenhuys et al., 2008). However, it has been demonstrated that bilateral receptive fields (RFs) in the somatosensory cortex (SC) are related not only to the body midline (e.g., oral cavity, the head, or the trunk) but also to the distal part of the extremities and in particular the hand (Geyer et al., 1999). The presence of neurons with bilateral RFs is reflected in the activation of both hemispheres during unilateral stimulation in both animals (Iwamura, 2000; Ruben et al., 2001; Iwamura et al.,

Correspondence should be addressed Henrik Jörntell, Neural Basis of Sensorimotor Control, Department of Experimental Medical Science, Lund University, SE-221 84 Lund, Sweden. E-mail: henrik.jorntell@med.Iu.se. DOI:10.1523/JNEUROSCI.2891-17.2018

Copyright $\odot 2018$ the authors $\quad 0270-6474 / 18 / 383669-11 \$ 15.00 / 0$ 
2002; Ferezou et al., 2007) and humans (Schnitzler et al., 1995; Zhu et al., 2007; Tamè et al., 2015).

Recent studies have indicated the existence of extensive bilateral integration of information in the brain. Indeed, haptic signals from the two hands are directly coupled, such that tactile and kinesthetic signals are combined as if they came from the same hand (Dupin et al., 2015). Moreover, unilateral stroke in cortex affects the tactile perception of sensory stimulation to both hands (Brasil-Neto and de Lima, 2008), and in rats bilateral activation of the SC appears necessary to solve a whisker discrimination task (Shuler et al., 2002). Hence, investigating how bilateral information is represented in the neuronal networks would be crucial to understand how the brain draws advantage of the bilateral inputs.

However, whereas multiple studies so far have been conducted to demonstrate the presence of bilateral inputs, for example in the secondary somatosensory areas of the monkey (Iwamura et al., 1994) and in the primary SC of the rat (Pidoux and Verley, 1979; Armstrong-James and George, 1988; Shuler et al., 2001; Tutunculer et al., 2006), most of the processing-related issues that have been addressed have been on arbitrary interactions between single-pulse inputs from the two sides (Shuler et al., 2001; Moxon et al., 2008). But the issue of to what extent the local neuronal circuitry contains detailed information about the nature of the tactile input from the ipsilateral (IL) side has not been explored.

To be able to quantify whether the activity of neurons carries any information regarding this "what" component of the tactile input, one needs a number of diversified inputs that each has a high degree of reproducibility. We previously introduced a method to deliver such diversified, reproducible spatiotemporal input patterns of low-intensity electrical activation of tactile afferents in local digit skin (Oddo et al., 2017). Indeed, for contralateral tactile input patterns, cells in the primary SC (S1) of the rat were shown to decode such tactile inputs with high accuracy (Oddo et al., 2017). Here, we wanted to explore whether neurons in S1 are able to decode, or to identify, tactile input patterns delivered to distal digit 2 skin also of the ipsilateral paw.

\section{Materials and Methods}

\section{Surgical procedures}

Adult male Sprague Dawley rats $(N=23$; weight, 279-380 g; male sex) were prepared for acute recordings by the following steps: (1) isoflurane sedation of the animal; (2) intraperitoneal injection ( $40 \mathrm{mg} / \mathrm{kg}$ ketamine, $4 \mathrm{mg} / \mathrm{kg}$ xylazine); (3) incision in the inguinal area (approximately onehalf inch, $12 \mathrm{~mm}$ ) along the natural angle of the hindleg; (4) separating the connective tissue to expose the femoral vein; (5) small incision in the vein and insertion of the catheter, whereafter a continuous infusion of the anesthetics $(\sim 5 \mathrm{mg} / \mathrm{kg} / \mathrm{h}$, ketamine plus xylazine in a 10:1 ratio) began. Subsequent surgery consisted in the exposure of the SC of the right side by opening a window in the skull (size, $2 \times 2 \mathrm{~mm}$; Fig. $1 A$ ). An electrocorticography electrode was positioned in the vicinity of the recording area and was used to monitor the irregular occurrences of sleep spindles, which are a sign of deep sleep, throughout the experiments (Schomer and Da Silva, 2012). To further evaluate the proper level of anesthesia, the absence of withdrawal reflexes to noxious pinch to the hindpaw was continuously checked. A layer of agarose was then put over the skull opening to protect the exposed cortex from dehydration and to increase the local mechanical stability of the brain tissue. At the end of the experiment, animals were killed with an overdose of barbiturate.

All animal experiment procedures in the present study were performed in accordance with institutional guidelines and were approved in advance by the Local Animal Ethics Committee of Malmö-Lund, Sweden (permit ID M118-13).

\section{In vivo recordings}

All recordings were made in vivo in the region of the SC. Patch-clamp pipettes were pulled from borosilicate glass capillaries using a Sutter
Instruments P-97 horizontal puller. The composition of the electrolyte solution in the patch pipettes was as follows (in $\mathrm{mM}$ ): potassiumgluconate 135, HEPES 10, $\mathrm{KCl} 6.0, \mathrm{Mg}$-ATP 2, and EGTA 10. The solution was titrated to $\mathrm{pH} 7.35-7.40$ using $1 \mathrm{M} \mathrm{KOH}$. The impedances of the patch pipettes were $8-24 \mathrm{M} \Omega$.

In each recording session, two pipettes were inserted into the exposed cortex and the signal was recorded using an EPC-800 patch-clamp amplifier (HEKA Elektronik) in the current-clamp mode (amplification gain of 50 times, with a bandwidth from DC to $100 \mathrm{kHz}$ ). After the insertion, the focus of the field potential (FP) was estimated by delivering single-pulse electrical tactile afferent stimulation to the contralateral second forepaw digit. If the elicited FP was of low amplitude $(<0.2 \mathrm{mV})$, the pipettes were removed and relocated in the brain.

The experiments consisted of two stages: (1) identification of neurons and (2) running the recording session. During stage 1, single-pulse stimulation (one pulse per second) was delivered to elicit local FPs. The pipette was moved slowly $(\sim 0.3 \mu \mathrm{m} / \mathrm{s})$ through the neocortex until the single-pulse stimulation evoked neuronal spikes. If the identified neuron displayed a good signal quality $(>3 \mathrm{mV})$ and stable activity (i.e., the spontaneous activity of the neuron remained within the same range \pm 5 $\mathrm{Hz}$ on a minute-by-minute basis), it was considered for the recording session. In the recording session, the activity of the neuron was continuously recorded and the preset tactile afferent stimulation protocol (see below) was launched. Only extracellular recordings were considered in this study.

All data were digitized at $100 \mathrm{kHz}$ using CED $1401 \mathrm{mk} 2$ hardware and Spike2 software (CED). Spike signals were analyzed off-line using inhouse software that identified the shape of the spike using a templatebased system, verifying that the overall shape of each neuron spike recorded remained the same for the duration of the recording. The recording depth from the surface of the brain for each neuron was noted.

\section{Electrical tactile stimulation}

The second forepaw digits of both sides were stimulated electrically (DS3 Isolated Stimulator, Digitimer; Fig. 1A). Stimulation of the left side (i.e., the side being opposite to the recording side, generated the CL responses), whereas the stimulation of the right side; i.e., same side of the recording site, generated the IL response (Fig. 1A).

We used eight different stimulation sequences (labeled F5, S5, F10, $\mathrm{S} 10, \mathrm{~F} 20, \mathrm{~S} 20, \mathrm{~F} \infty$, and $\mathrm{S} \infty$; Fig. $1 B$, top panels), which were the same predefined spatiotemporal patterns we used previously (Oddo et al., 2017). In that previous study, these spatiotemporal patterns were obtained from an artificial fingertip by repeatedly moving it against four probes of different shapes using a cyclic motor. The resulting mechanical forces were transduced into receptor potentials in four sensors of the artificial fingertip, and these receptor potentials were converted to spike trains using either a fast or a slow adaptation of the spike generator to generate different types of spike output from the sensors. The artificial fingertip provided the advantage that it synthesized spatiotemporal patterns of skin sensor activation at quasi-natural rates that follow a natural overall temporal modulation, or "envelope," that the biological lowthreshold mechanoreceptors of the skin are known to display under dynamic indentation. Of the many spatiotemporal spike output patterns recorded from the neuromorphic sensors (one per repetition), we selected two patterns for each of the four indentation probes used. These eight patterns were characterized by a high resolvability (Oddo et al., 2017), thereby presumably facilitating the decoding performance subsequently detected in the spike responses of the neocortical neurons. These eight spatiotemporal patterns of multichannel electrical skin site activation were used throughout the experimental series, and were delivered to the second digits of the two forepaws through separate channels. Briefly, four pairs of needle electrodes were inserted intracutaneously into predetermined skin sites of the second digit of each forepaw (Fig. 1A, group of Ch1-Ch2-Ch3-Ch4 for the left forepaw, group of Ch5-Ch6-Ch7-Ch8 for the right forepaw). The interneedle distance for each pair of bipolar electrodes was 2-3 mm. Each needle pair delivered a single-pulse stimulation (intensity, $0.5 \mathrm{~mA}$; pulse duration, $0.14 \mathrm{~ms}$ ), and the combination of multiple pulses across time and channels constituted the specific spatiotemporal tactile inputs (for further details, see Oddo et al., 2017). 
A

B
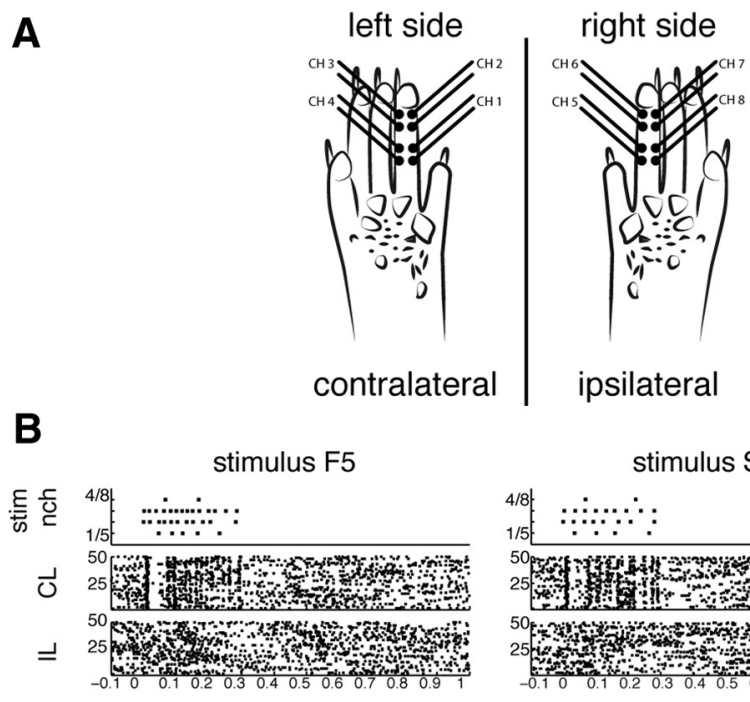

stimulus F20

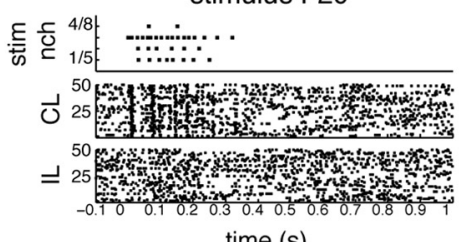

C
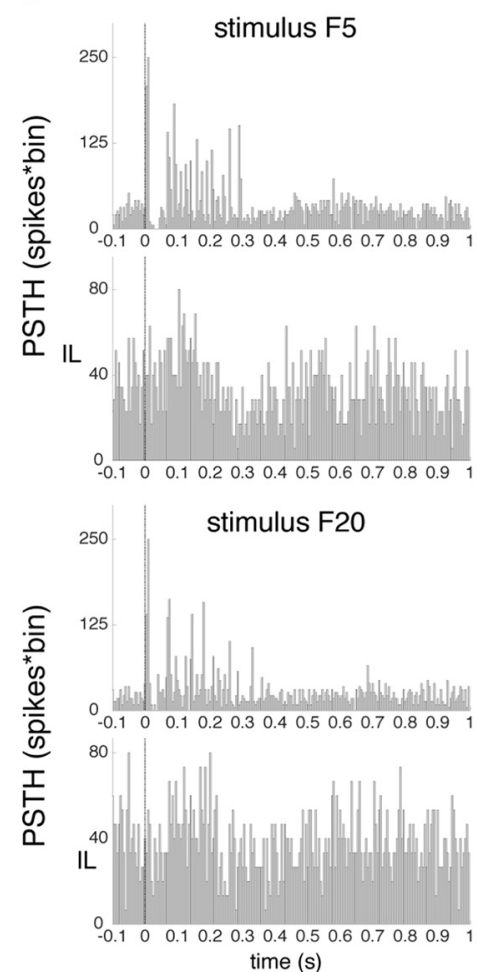

stimulus S5

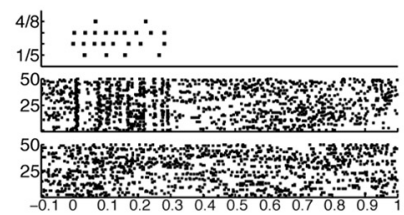

stimulus $\mathrm{S} 20$

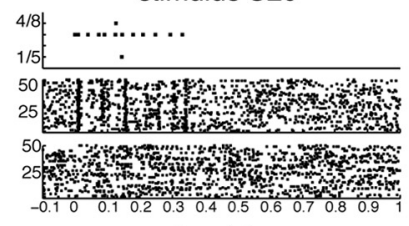

time (s)
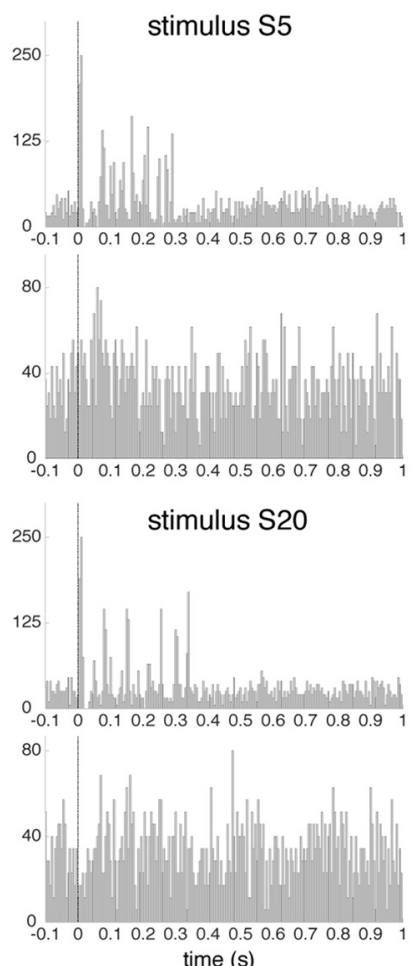

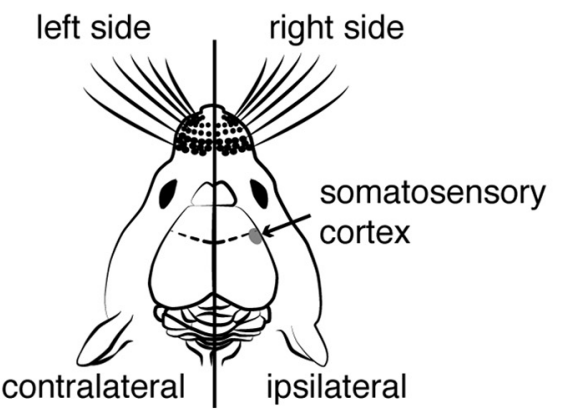

stimulus F10
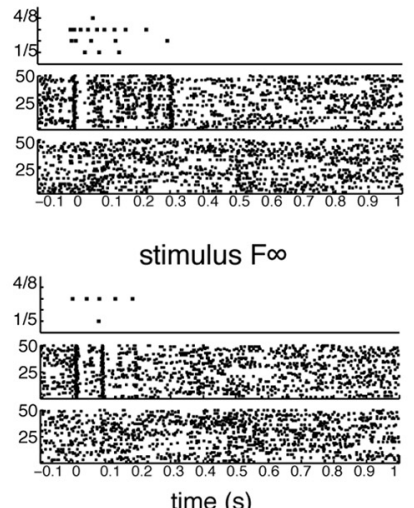

time (s)
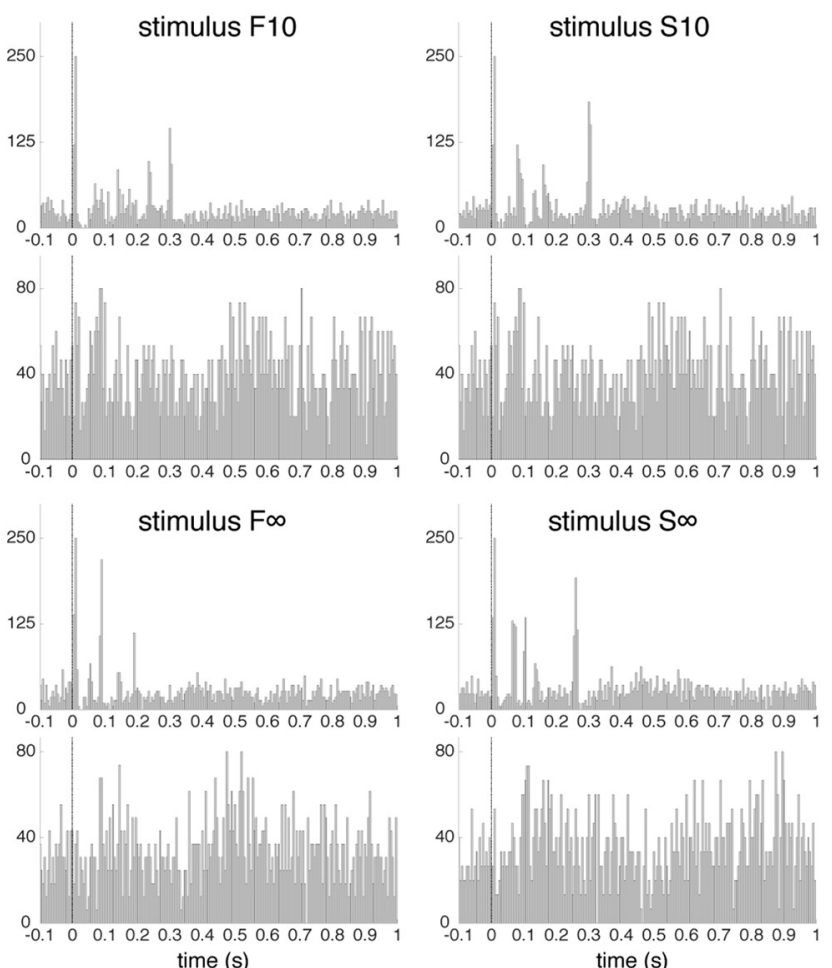

Figure 1. Neuronal spike responses during bilateral stimulation of the second digits. $A$, The activity of neocortical neurons was recorded in the right somatosensory cortex of rats via the patch-clamp technique. Eight stimulation patterns (labelled F5, S5, F10, S10 F20, S20, F $\infty$, and S $\infty$ (for further details, see 0 ddo et al., 2017) were delivered via eight pairs of intracutaneous needles (or channels; $\mathrm{CH} 1, \mathrm{CH} 2, \mathrm{CH} 3$, and $\mathrm{CH} 4$ for the $\mathrm{CL}$ side; $\mathrm{CH} 5, \mathrm{CH} 6, \mathrm{CH} 7$, and $\mathrm{CH} 8$ for the IL side) positioned in the second digit of the right and left forepaws. The contralateral response was elicited by stimulating the left side (opposite to the recording side), whereas the ipsilateral response was generated by stimulating the right side (same side as the recording side). $\boldsymbol{B}$, The spatiotemporal sequence of the tactile stimulus [top panel indicated as "stim nch" (i.e., stimulation of channel number N)], the CL responses and the IL responses. The evoked neuronal spike responses for the time window [ -0.11$]$ s are shown as raster plots (where each dot indicates the time of occurrence of a spike, with the responses for all trials being represented in the display). C, The CL and IL PSTHs of the same sample neuron for each stimulation pattern. F, Fast adaptation; $S$, slow adaptation. 


\section{Experimental design}

The spatiotemporal tactile input patterns were delivered to the skin of the contralateral and ipsilateral digit 2 of the forepaws in sequence. Each tactile stimulus was repeated 50 times per digit (50 repetitions $\times 8$ patterns $\times 2$ digits $=800$ repetitions in total). A pseudorandom order was adopted for delivering the stimulation patterns to avoid temporal and side dependency across repetitions. Each stimulation pattern lasted for $270-340 \mathrm{~ms}$, whereas the time interval between two consecutive patterns was set to $1.8 \mathrm{~s}$ to allow a relaxation of the cortical activity induced by the stimulation. In this experiment, a set of 13 animals was used to obtain a total of 67 recorded neurons.

An additional experiment was performed during which a bilateral stimulation (i.e., delivering the CL and IL stimulation patterns of each type in synchrony; $N=5$ animals to obtain $N=13$ neurons) was added to the protocol described above (which hence was extended to 50 repetitions $\times 8$ patterns $\times 3$ digit combinations $=1200$ repetitions in total).

\section{Statistical analysis}

Characterization of the single neuron response latencies and amplitudes. The raw neural signals were processed to perform a spike detection analysis in the time window of $[-0.21 .0]$ s (i.e., from $0.2 \mathrm{~s}$ before the stimulation onset to $1.0 \mathrm{~s}$ after). The time of $0 \mathrm{~s}$ indicated the stimulus onset, whereas the spike activity in $[-0.20]$ s represented the baseline activity.

Per each stimulation and each trial, neural spikes were visualized via raster plots. Subsequently, peristimulus time histograms (PSTHs) were computed to visualize the time and rate of the neural spiking discharge for each stimulation pattern (bin width set to $5 \mathrm{~ms}$ ). The basic characterization of the neuronal responses consisted of their amplitudes and the response latency times. The response amplitude was quantified from the PSTH as the bar with the highest value after the stimulation onset (Fig. $2 A, B$, arrows), expressed as the number of SDs from the baseline (Fig. $2 C$ ). The response latency time was computed after applying an optimized kernel density estimation (KDE; Shimazaki and Shinomoto, 2010) and was defined as the temporal instant of the maximum value of the estimated neural responses (Fig. 2A, B, dots).

The distribution of the amplitude and latency in the neuron population across tactile stimuli and stimulation sides were evaluated by the grand average (GA; i.e., the mean of all the neurons) as well as the SE to monitor the variability in the sample group. The differences between neurons and between stimulation sides were evaluated using a two-way ANOVA (significance threshold, 5\%).

Decoding analysis. The decoding performance for the spatiotemporal tactile inputs based on the spiking responses of single cortical neurons was calculated by using principal component analysis (PCA; for review, see Oddo et al., 2017). This analysis answered the question of by what precision the spiking response could be used to distinguish one stimulation pattern from the others in each individual neuron. We decoded the stimuli from the spiking patterns of single cortical neurons using an improved and simplified version of a method based on the decomposition of the temporal profiles of the evoked spike responses into principal components (PCs), as follows: (1) we computed the mean firing rate over time in the response to each stimulus presentation; (2) we converted the spike trains evoked by each stimulus presentation into continuous functions by convolving them with an exponential kernel with a characteristic time of $5 \mathrm{~ms}$; (3) we computed the average of the resulting functions for each stimulation pattern to obtain a template of the average temporal evolution of the response to each stimulus; (4) to highlight the differences in the temporal profile, we $z$-scored the average responses (Fig. $3 A$, examples); (5) we extracted the PCs of the $z$-scored average responses evoked by the different stimulation patterns, and we computed for each individual response the score relative to each PC, that is, the scalar product between the response temporal vector and the PC temporal vector; (6) the decoding was performed using the mean firing rate and the scores on each of the first 7 PCs for each individual response, and the average intensity and the temporal profile of the individual response hence determined the location of that response in this eight-dimensional space; and (7) to decode the stimuli from the response patterns, we used the k-NN (knearest neighbor) classification procedure, as previously described (Quian Quiroga and Panzeri, 2009). As stated in the previous point, the
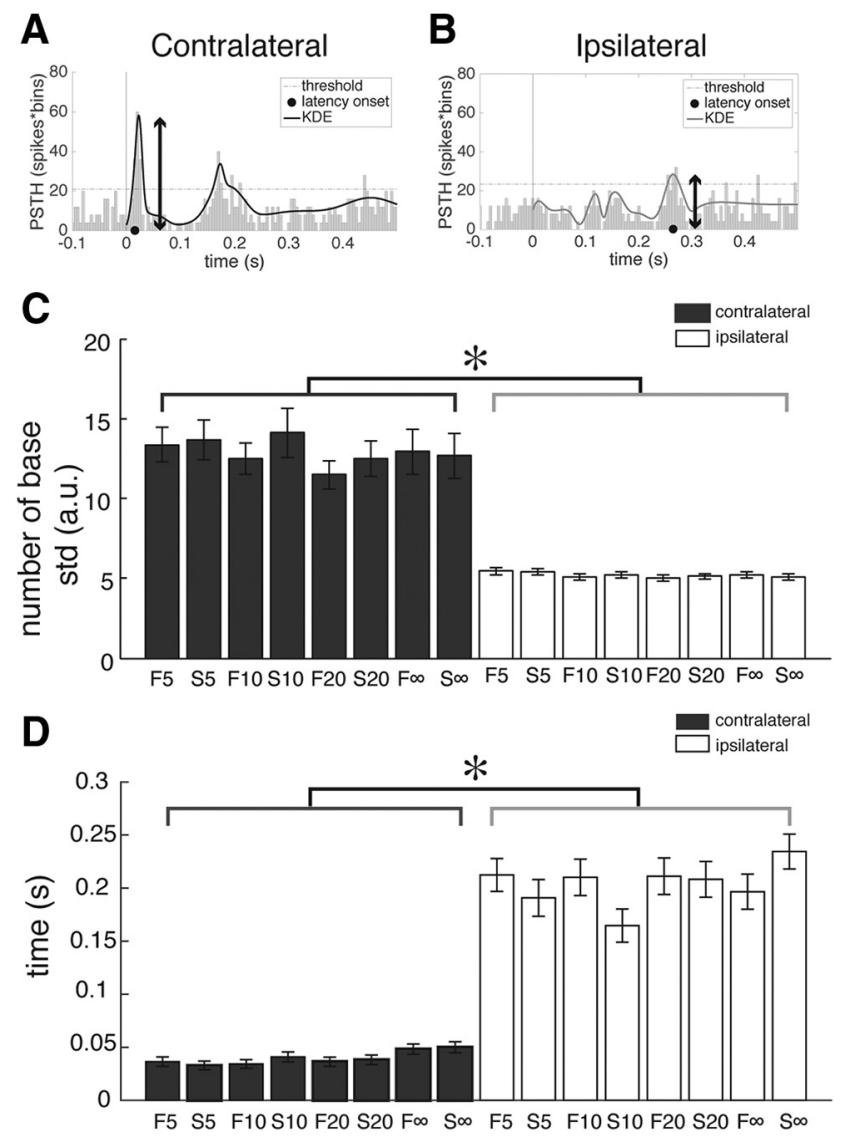

Figure 2. Characterization of the spike responses to $C L$ and IL stimulations. $A, B$, Illustration of one example of PSTHs each for a CL and an IL stimulation pattern (pattern F5 in both cases) overlapped to the estimates obtained by applying an optimized KDE. Two features were computed for each neuron, the maximal amplitude from the PSTH ( $\boldsymbol{A}$ and $\boldsymbol{B}$, arrows) and latency onset as the temporal instant of the maximum value of the $\operatorname{KDE}(\boldsymbol{A}$ and $\boldsymbol{B}$, dots). The dashed line is the baseline threshold (see Materials and Methods). C, Amplitude distribution across stimuli measured in the number of SDs from the baseline (indicated as the number of base SDs). Statistical differences were found between responses evoked from the two different digits $\left(F_{(1,1056)}=311.76,{ }^{*} p<0.00001\right.$, two-way ANOVA) but not between responses to stimuli applied to the same digit $\left(F_{(7,1056)}=0.58, p>0.05\right.$ for both $\mathrm{CL}$ and IL sides, two-way ANOVA). $D$, The CL onset latency was significantly shorter than for IL stimuli $\left(F_{(1,1056)}=728.57,{ }^{*} p<\right.$ 0.001 , two-way ANOVA), whereas no significant effect was found between stimuli applied to the same digit $\left(F_{(7,1056)}=1.73, p>0.05\right.$, two-way ANOVA). F, Fast adaptation; S, slow adaptation.

response to each stimulus presentation was represented as a point in a space with eight dimensions. Half of these points were selected at random as the training set, and the other half was used as the test set. For each trial belonging to the test set, we identified the closest nine trials in the training set with a Euclidean distance in the eight-dimensional space. The trial was then classified as elicited by the same stimulus that elicited the relative majority of the nine neighbors. We performed 40 iterations of the decoding, each with different training and test sets, and we averaged the fraction of correctly decoded trials in each iteration to get the decoding average of the neuron. We also computed and averaged over all iterations the confusion matrix information. The confusion matrices represent (column wise) the computed decoding per each presented stimulus. Each column represented the simulation class, and thus the correct responses are reported in the diagonal (Fig. 3B). The mean decoding was thus defined as the average of the diagonal in the confusion matrix. The decoding analysis (i.e., segregation of single neuron responses and computation of the mean decoding) was also performed for the synchronous (SYN) data. The statistical difference among CL, IL, and SYN mean decoding were evaluated by applying the nonparametric Kruskal-Wallis test (for results, see Fig. 6). 
A
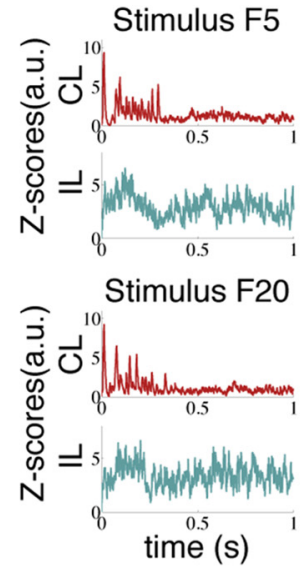

B

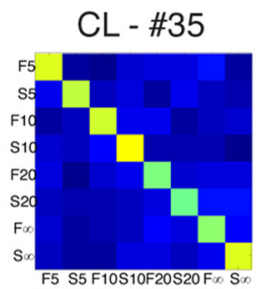

$\mathrm{CL}-\# 59$
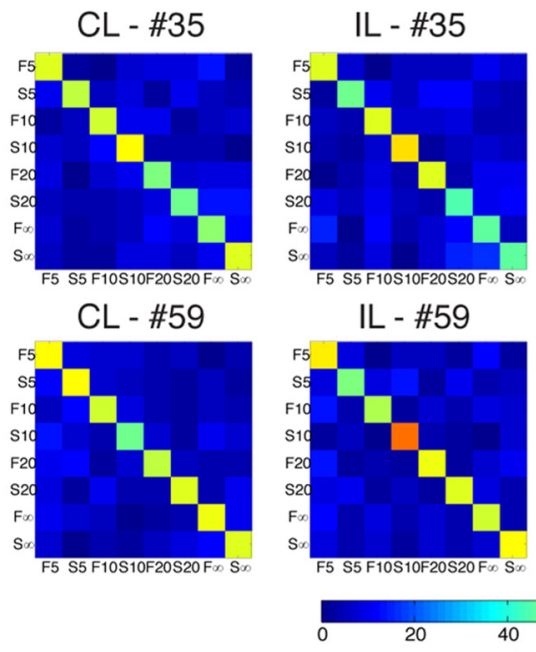

C

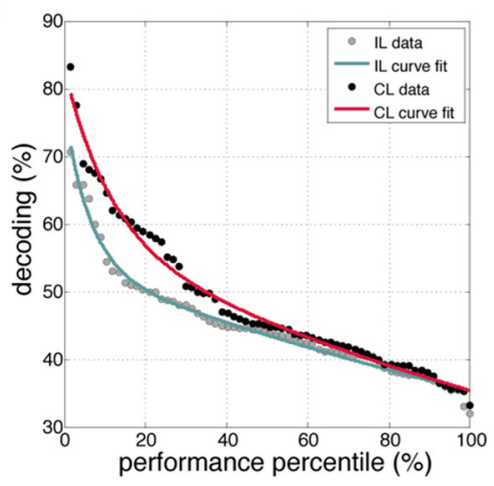

IL - \#59

Stimulus S5
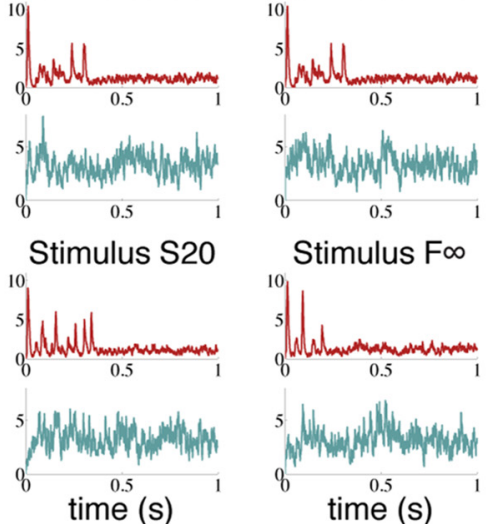

CL - \#58

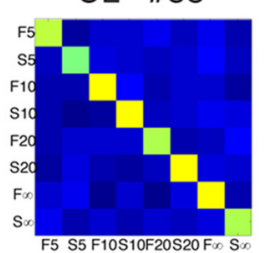

CL - \#66
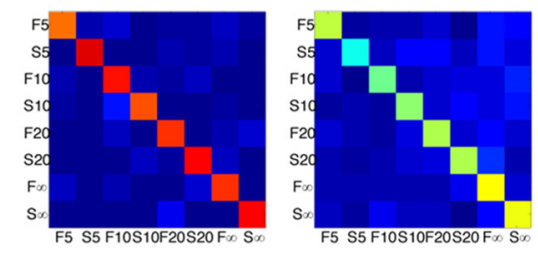

IL - \#66
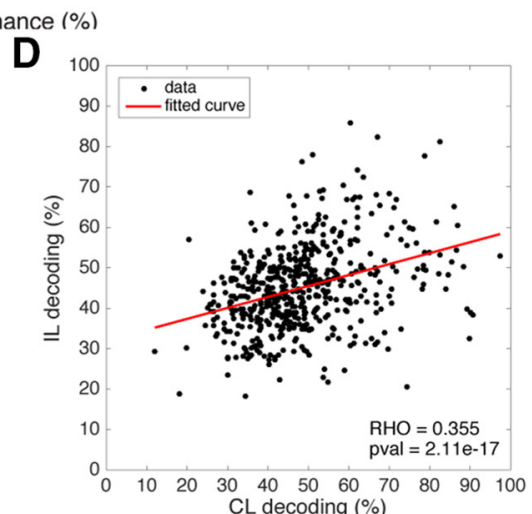

Figure 3. Single neuron decoding analysis. $\boldsymbol{A}$, Input-specific time evolutions of the spike responses illustrated by the timecontinuous z-score functions for all eight stimulation patterns delivered to the second digit on the two sides. The $z$-scores were used to normalize the amplitudes of each trace, thus facilitating comparisons of the temporal evolutions of the response intensity. $B$, Confusion matrices illustrating the decoding performance for the $C L$ and IL input patterns for a set of representative neurons. $C$, Distribution of decoding performance across neurons and corresponding curve fits for the $C L$ and IL decoding. $D$, The IL and CL decoding values per each stimulation and neuron. Pearson correlation showed a weak but significant correlation ( $p=2.11 \mathrm{e}-17)$. F, Fast adaptation; S, slow adaptation.

To display a summary of the results, the CL and IL mean decoding values were also sorted in a descending order to visualize the data distribution across neurons. The sorted data were then fitted by exponential curves by using the following exponential model:

$$
y(n)=a_{1} * \exp \left(-n / b_{1}\right)+a_{2} * \exp \left(-n / b_{2}\right)
$$

where $a_{1}$ and $a_{2}$ are the amplitude of each exponential, $b_{1}$ and $b_{2}$ are the decays of each exponential, and $n$ is the neuron number (in the sorted order).
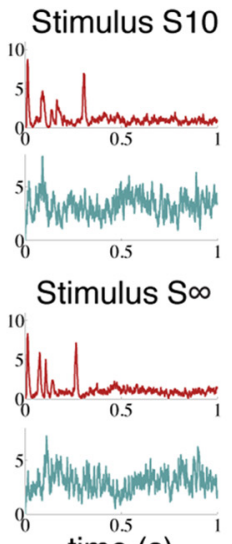

time (s)

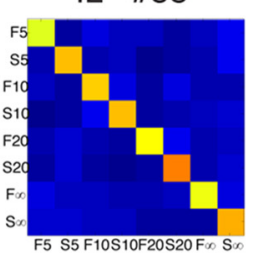

The correlation between CL and IL decoding values across stimuli and neurons was evaluated by plotting the CL and IL decoding in the $x$ - and $y$-planes, respectively. This correlation was then quantified by computing the Pearson correlation (reporting $p$ and $\rho$ values).

Relationship between neuron depth and decoding. The relationship between the depth of the recorded neurons and the decoding performance was tested using Pearson linear correlation analysis and Kruskal-Wallis test.

Time-dependent analysis of the mean decoding. In addition to the standard time window of $1.0 \mathrm{~s}$ cited above, we evaluated the dependency of the decoding on the total time integration window considered. This part of the decoding analysis was performed in the following $10 \mathrm{sep}$ arate integration time windows: $\left[\begin{array}{ll}0 & 0.1\end{array}\right] \mathrm{s},[0$ $0.2] \mathrm{s},\left[\begin{array}{ll}0 & 0.3\end{array}\right] \mathrm{s},\left[\begin{array}{ll}0 & 0.4\end{array}\right] \mathrm{s},\left[\begin{array}{ll}0 & 0.5\end{array}\right] \mathrm{s},\left[\begin{array}{ll}0 & 0.6\end{array}\right] \mathrm{s},\left[\begin{array}{ll}0 & 0.7\end{array}\right]$, $\left[\begin{array}{ll}0 & 0.8\end{array}\right]$ s, $\left[\begin{array}{ll}0 & 0.9\end{array}\right]$ s, and [ $\left[\begin{array}{ll}0 & 1\end{array}\right]$ s. The group decoding in each temporal window was reported by computing the GA (i.e., the average of the mean decoding across all the neurons). The statistical difference between the CL and IL GA across the integration time windows was evaluated by applying the nonparametric Wilcoxon rank sum test.

Information theory of neural responses. The information theory analysis quantified the discrimination of neural responses among different stimuli by evaluating the trial-by-trial variability (Magri et al., 2009; Ince et al., 2010; Panzeri et al., 2010). In this study, the chosen neural response was the firing rate, computed as follows:

$$
r=\frac{n}{T},
$$

where $n$ is the number of spikes in the temporal windows $t_{1} \leq t \leq t_{2}$ and $T=t_{2}-t_{1}$.

The mutual information (MI) was estimated per each neuron separately for the contralateral and ipsilateral responses by using the Information Breakdown ToolBox (ibTB; Magri et al., 2009). The data fit the Gaussian distribution, and therefore the Gaussian method of ibTB together with the related bias correction was used.

The mutual information was computed as $\mathrm{I}([T-0.1, T],[0, T-0.1])-\mathrm{I}([0, T-0.1])$, $T \in[0.1: 0.1: 1]$ s to evaluate the nonredundant information content across time. Indeed, each temporal window was adjusted for the contribution of the previous interval, and thus the computed values represent the number of new contributions to the tactile information content for that specific temporal window. For each temporal window, the GA (i.e., the mean of the MI across neurons) was reported.

\section{Results}

Neural responses during contralateral and ipsilateral stimulation

This study aimed to describe the neuronal responses evoked by contralateral and ipsilateral stimulation of the second digit of both forepaws and to investigate the decoding of such inputs at the single neuron level. In the right primary SC of the rat, we recorded from 67 neocortical neurons extracellularly using the 
loose-patch cell-attached recording technique while delivering electrical tactile inputs through intracutaneous needles inserted into the second digit of the left (i.e., CL) and right (i.e., IL) forepaws (Fig. 1A). The tactile stimuli (Fig. $1 B$, top, stimulation pulses) were eight different multichannel patterns previously generated using a set of neuromorphic pressure sensors (same patterns as in the study by Oddo et al., 2017) and delivered alternatively to the contralateral and ipsilateral sides in a pseudorandom sequence.

As a rule, robust neural responses were recorded for each stimulation pattern delivered to the contralateral digit, whereas responses to the ipsilateral stimulation patterns were weaker, as reported in the raster plots of a representative neuron (Fig. 1B, CL and IL responses). However, in the average responses, which were obtained by computing the PSTH across trials, the IL responses became more evident (Fig. 1C). The CL stimulation induced stimulus-dependent and sharp responses with a discriminable temporal structure, whereas the ipsilateral responses were less reliable but still displayed appreciable variability of the temporal profiles across stimuli (Fig. 1C). Furthermore, the strongest CLevoked activity occurred during the ongoing delivery of each stimulation sequence (up to $0.34 \mathrm{~s}$, compare Fig. $1 B$ ), whereas the IL responses could often peak after the end of the stimulation sequence (Fig. 1C, peak of F10 between 0.5 and $0.6 \mathrm{~s}$ ).

\section{Characterization of the magnitude and response latencies of the evoked spike activity}

The CL and IL PSTHs and corresponding KDEs were computed for the temporal window -0.2 to $1.0([-0.21] \mathrm{s})$ to illustrate the temporal evolution of the neural responses. The PSTHs were used to extract the maximal amplitude (Fig. 2A,B, arrows), whereas the KDEs were used to calculate the response onset latency time (Fig. 2A, B, dots; for further details, see Materials and Methods).

The amplitudes of the responses evoked by the eight different CL input patterns were not statistically different and the same was true for the response amplitudes for the IL input patterns $\left(F_{(7,1056)}=0.58, p>0.05\right.$, two-way ANOVA; Fig. $\left.2 C\right)$. However, the variability of the CL response amplitudes (amps) was higher than that for IL (CL amp [min, $\max ]=[5,35]$ a.u.; IL amp [ minimum, maximum $]=[2,8]$ a.u.). As a group, the CL response amplitudes (mean \pm SE, $12.9 \pm 1.2$ a.u.) were significantly higher than the IL response amplitudes (mean $\pm \mathrm{SE}, 5.2 \pm 0.2$ a.u.; $F_{(1,1056)}=311.76, p<0.001$, two-way ANOVA).

The response latency times for the IL input patterns were very long and had a high variability (mean $\pm \mathrm{SE}, 0.206 \pm 0.021 \mathrm{~s}$ ), whereas the latency times of the CL responses were short (mean \pm $\mathrm{SE}, 0.040 \pm 0.006 \mathrm{~s})$. The IL and CL latency times were statistically different $\left(F_{(1,1056)}=728.57, p<0.001\right.$, two-way ANOVA; Fig. $2 D)$. However, the differences among the eight stimulation patterns within each respective group were not statistically different $\left(F_{(7,1056)}=1.73, p>0.05\right.$, two-way ANOVA; Fig. $\left.2 D\right)$.

Hence, the magnitude and latency of CL responses were clearly different from those evoked by the IL stimulation. The much longer latencies of the IL responses suggested that they were generated by more indirect pathways, possibly with less dominance of direct cuneo-thalamo-cortical pathways, than for the CL responses. Note, however, that the purpose of our study was not to identify the shortest possible latency times for the IL/CL inputs-indeed, we deliberately kept the stimulus intensities low, as our main interest was the responses to the patterned stimuli, which can be expected to result in longer response latency times because of the fewer afferent fibers activated.
Table 1. Curve fit parameters for the fast and slow exponential decays of the decoding in the temporal window $\left[\begin{array}{ll}0 & 1\end{array}\right] \mathrm{s}$ across the neuronal population

\begin{tabular}{|c|c|c|c|c|}
\hline & \multicolumn{2}{|c|}{$\begin{array}{l}\text { Fast decay exponential } \\
\left(a_{1}, b_{1}\right)\end{array}$} & \multicolumn{2}{|c|}{$\begin{array}{l}\text { Slow decay exponential } \\
\left(a_{2}, b_{2}\right)\end{array}$} \\
\hline & Contralateral & Ipsilateral & Contralateral & Ipsilateral \\
\hline Performance perception decay (\%) & 11 & 6 & 202 & 240 \\
\hline Decoding amplitude (\%) & 25 & 23 & 58 & 54 \\
\hline
\end{tabular}

\section{Neuron decoding analysis with contralateral and ipsilateral stimulation}

Both IL and CL stimulation patterns generated input-specific time evolutions of the spike responses (Fig. $3 A$ for the same example neuron as in Fig. 1), which is a requirement for decoding. We have previously demonstrated that single S1 neurons can effectively decode the same CL stimulation patterns as we used here (Oddo et al., 2017). Here, the goal was to investigate whether S1 neurons could decode the same patterned stimuli delivered to the ipsilateral side and whether there was a relationship between such decoding for inputs from the two sides. The decoding analysis was performed in the temporal window [ $\left[\begin{array}{ll}0 & 1\end{array}\right]$ s.

Representative examples of CL and IL decoding are reported in Figure 3B. These neurons were able to segregate both CL and IL inputs with very high precision, but the correlation between the CL and IL decoding varied (Fig. 3B). Indeed, neurons 35 and 59 showed very similar CL and IL decoding (mean decoding: CL$35=55 \%$ and IL-35 $=53 \%$; CL-59 $=58 \%$ and IL-59 $=59 \%$ ), whereas neuron 58 showed higher IL decoding values (CL-58 = $57 \%$ and IL-58 $=66 \%$ ); and for neuron 66 , the decoding of the CL input was higher (CL-66 $=82 \%$ and IL-66 $=53 \%$ ).

The sorted CL and IL mean decoding for the population of recorded neurons is reported in Figure 3C. Individual neurons could decode the CL tactile information with high accuracy (maximum value, $>80 \%$ ) as well as the IL input (maximum value, $>70 \%)$. In general, the CL decoding was slightly higher than the IL decoding (mean \pm SD: $C L=48 \pm 11 \%$; IL $=45 \pm$ $8 \%)$. The curve fits for the distributions of the CL and IL decoding levels were very similar even if the CL curve was located at slightly higher levels than the IL curve (Fig. $3 C$, red and blue lines). Regarding the estimated parameters of the fitting curves reported in Table 1, the IL exponential decayed more rapidly than the CL exponential in the fast decay part of the exponential curves, whereas the CL and IL curves overlapped in their slow decay.

Finally, the correlation between CL and IL decoding was computed as the Pearson correlation of the mean decoding (Fig. 3D). The results showed a very weak, but significant, linear correlation $(\rho=0.384, p=3.02 \mathrm{e}-20)$. Therefore, there was only a weak relationship between the CL and IL decoding in the individual neurons.

Hence, the neuronal responses generated by the IL stimulation patterns appeared to carry almost as much information about the "what" (or type) of the tactile input as the responses to the CL stimulation patterns, at least in the evaluated temporal window (i.e., $\left.\left[\begin{array}{ll}0 & 1\end{array}\right] \mathrm{s}\right)$.

In another, larger material of S1 neurons, we have previously reported an absence of correlation between recording depth and decoding of CL inputs (Oddo et al., 2017). In the present material, we found a weak but significant positive correlation between recording depth and decoding for both CL inputs ( $\rho=0.358, p=$ $0.003)$ and IL inputs $(\rho=0.258, p=0.04)$. We believe that the difference is due to the smaller sample size of the present material and that the conclusion of our previous study, that there is no 
A

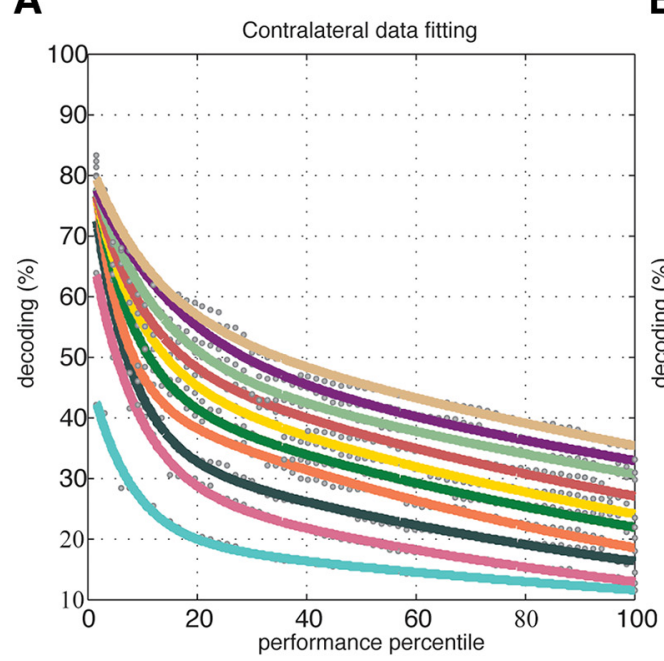

B

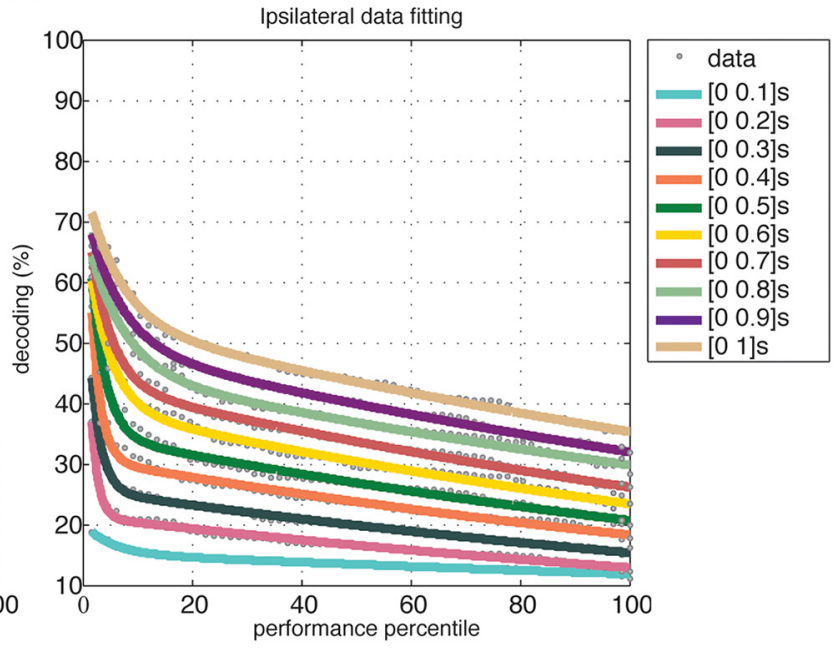

C

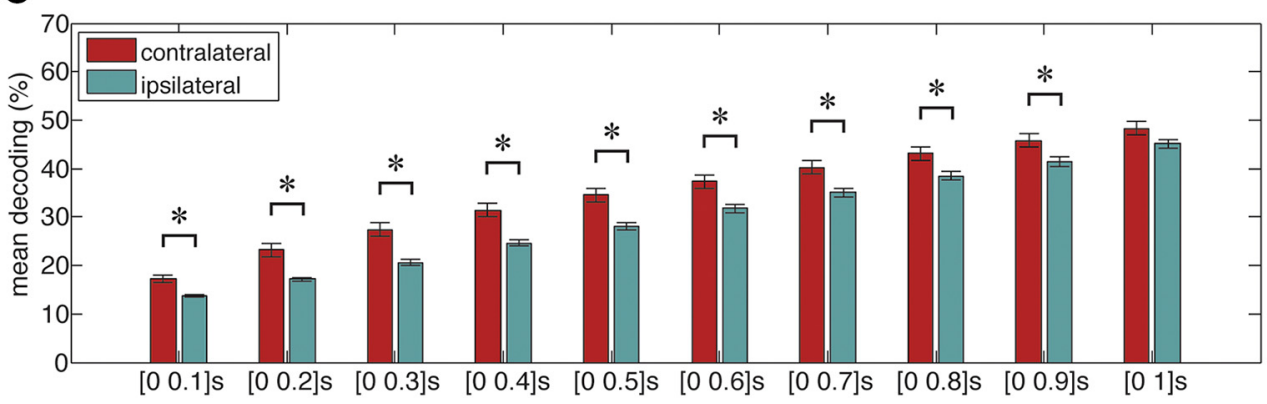

Figure 4. Temporal evolution of the decoding performance. $\boldsymbol{A}$, Distribution of the decoding performance across the neurons, and the corresponding curve fits, for the 10 integration time windows for the $\mathrm{CL}$ stimulations. $\boldsymbol{B}$, Corresponding plot for the IL stimulations. C, The grand average (i.e., mean of the decoding of all neurons) for the $\mathrm{CL}$ (red) and the IL (blue) stimulations for different integration time windows. The statistical differences between the $\mathrm{CL}$ and IL decoding were significant for all groups except the last integration time window ( ${ }^{*} p<0.05$, Wilcoxon rank sum test).

relationship between recording depth and decoding, is more reliable.

\section{Tactile information over different time windows}

Integration of tactile information between hemispheres is believed to occur already at short latency times (Tamè et al., 2015). Therefore, we next investigated the gradual temporal evolution (from 0 to $1 \mathrm{~s}$ after stimulus onset) of the decoding performance for 10 different integration time windows (starting from $0 \mathrm{~s}$ with incremental steps of $100 \mathrm{~ms}$ ).

For the mean decoding performance, the neuronal distributions are shown for the 10 different integration time windows together with the corresponding curve fits, with CL and IL decoding data shown separately (Fig. $4 A, B$, respectively). The CL curve fits (Fig. $4 A$ ) had a similar shape for all integration time windows, except for the shortest (i.e., [ $\left.\begin{array}{ll}0 & 0.1\end{array}\right]$ s), suggesting that most neurons that contributed with high decoding did so already from the first couple of $100 \mathrm{~ms}$ of the stimulus presentations, although the overall mean decoding improved with the duration of the integration time window.

Conversely, the IL curve fits illustrated a progressively increasing overall decoding with the duration of the integration time window (Fig. 4B). In particular for short integration time windows, the curve fits of the neuronal decoding distribution had a sharp corner at a low percentile, suggesting that very few of the neurons contributed with a high decoding performance, at least up to the time integration window of $400 \mathrm{~ms}$ ( $0.4 \mathrm{~s}$; Fig. $4 B$ ). However, for the longest integration time windows, the IL curve fit indicated a distribution that was more similar to the CL curve (Fig. 4A, B, [0 1]s; i.e., with a larger proportion of neurons contributing with higher decoding). The estimated values of each curve fit are reported in Table 2.

For both the CL and the IL stimuli, the GA (i.e., the average of all neurons) of the decoding performance increased approximately linearly with the integration time window (Fig. 4C). The CL decoding was higher than the IL decoding for short integration time windows (Fig. 4C, [ 00.1$]$ s and [ 00.2$] \mathrm{s}$ ), whereas the gap between the CL and IL decoding decreased after $0.8 \mathrm{~s}$ and was almost eliminated after $1.0 \mathrm{~s}$ (Fig. 4C). Indeed, the differences between CL and IL decoding performance were statistically different only up to the $0.9 \mathrm{~s}$ time

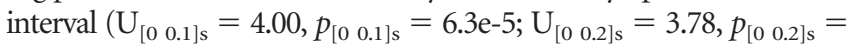

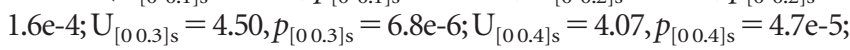

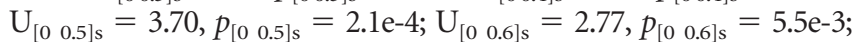

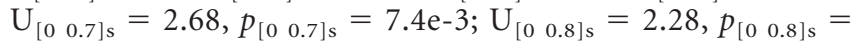

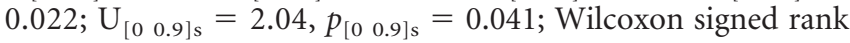
test), whereas for the full $1.0 \mathrm{~s}$ time window the computed $p$ value indicated that the difference was no longer significant $\left(U_{\left[\begin{array}{ll}0 & 1\end{array}\right] \mathrm{s}}=\right.$ $\left.1.45, p_{[0} 1\right] \mathrm{s}=0.147$, Wilcoxon signed rank test; Fig. $4 C$ ).

To complement the decoding analysis, the CL and IL mutual information is reported in Figure 5. The highest CL information content was found in the three time windows between 0.2 and $0.4 \mathrm{~s}$, whereas the IL information content was evenly distributed across all time windows. Hence, except for the temporal windows between 0.2 and $0.4 \mathrm{~s}$ (i.e., during the ongoing input patterns), the CL and IL mutual information was comparable across time (Fig. 5). 


\section{Neuronal decoding analysis for simultaneous bilateral stimulation}

Additional experiments were performed to investigate the decoding of simultaneous bilateral tactile afferent activation. In a separate group of animals, the same set of tactile input patterns was delivered to the CL side, to the IL side, as well as to the CL and IL sides in SYN (Fig. 6A-C). The SYN stimulation resulted in essentially the same decoding as for the CL stimulation alone. This is shown in Figure 6D, in which the two fitted curves for SYN and CL approximately overlapped, whereas the fitted curve for the IL stimulation was located at slightly lower values. The GA decoding values were similar for CL and SYN $(C L=52 \%$; SYN $=51.5 \%)$, whereas the IL was lower but still comparable (IL $=43.4 \%$; $\mathrm{H}(2)=3 ; p>0.05$, Kruskal-Wallis test; Fig. $6 E)$. Nevertheless, these results showed that there was no additional decoding effect of adding the IL input to the CL input when delivered in synchrony. Additionally, the similar values between the CL and the SYN decoding across the population (Fig. $6 E$ ) suggested a priority for processing contralateral inputs.

\section{Discussion}

Using single neuron recordings in the rat SC in vivo, we found that the decoding of a set of spatiotemporal patterns of tactile afferent stimulation delivered to the skin of the second digit reached comparable levels regardless of whether the input was delivered to the contralateral or the ipsilateral forepaw, given sufficient integration time. Against the background of previous evidence of bilateral signaling in neocortical somatosensory neurons, this was a surprising finding. In particular, that the neocortical neurons could decode IL inputs when there were such small differences between the patterns suggests that S1 cortex has access to very precise information about IL inputs.

\section{Comparable decoding of CL and IL tactile inputs in single neurons}

Although the responses to the CL stimulation patterns were much faster and larger than those evoked by the IL stimulation patterns (Figs. 1, 2), both the CL and IL inputs evoked responses with stimulus pattern-dependent temporal profiles (Fig. 3A). These differences in response profiles were evaluated using a PCAbased decoding analysis for each individual neuron (Fig. 3B), which surprisingly indicated that the decoding performances for the $\mathrm{CL}$ and IL responses were not very different (Fig. 3C). Notably, the decoding algorithm is powerful in the sense that it will identify systematic differences in response profiles between different stimulation patterns. It is not known to what extent the brain uses all of the information available at the single neuron level, but our findings indicate that the information would be present, available for the brain to use. The results also indicate that the more intense CL responses may not necessarily be associated with higher decoding. This could be explained if, despite the higher response intensity, there is no corresponding increase in response consistency. It could also happen if the signal-tonoise ratio is unaffected by response intensity. However, in terms of decoding time, the CL input was more rapidly decoded than the IL input (Figs. 4, 5), suggesting that CL input is still dominating in the local processing.
Each curve fit consisted of a fast and a slow decay exponential.

\begin{tabular}{|c|c|c|c|c|c|c|c|c|}
\hline \multirow{3}{*}{$\begin{array}{l}\text { Temporal } \\
\text { window }\end{array}$} & \multicolumn{4}{|c|}{ Fast decay exponential } & \multicolumn{4}{|c|}{ Slow decay exponential } \\
\hline & \multicolumn{2}{|c|}{$\begin{array}{l}\text { Decoding } \\
\text { amplitude } \\
(\%)\end{array}$} & \multicolumn{2}{|c|}{$\begin{array}{l}\text { Performance } \\
\text { perception } \\
\text { decay }(\%)\end{array}$} & \multicolumn{2}{|c|}{$\begin{array}{l}\text { Decoding } \\
\text { amplitude } \\
(\%)\end{array}$} & \multicolumn{2}{|c|}{$\begin{array}{l}\text { Performance } \\
\text { perception } \\
\text { decay (\%) }\end{array}$} \\
\hline & $\mathrm{CL}$ & IL & $\mathrm{CL}$ & IL & $\mathrm{CL}$ & IL & $\mathrm{CL}$ & IL \\
\hline$\left[\begin{array}{ll}0 & 0.1\end{array}\right] \mathrm{s}$ & 2 & 5 & 7 & 5 & 20 & 15 & 179 & 397 \\
\hline$\left[\begin{array}{lll}0 & 0.2\end{array}\right] \mathrm{s}$ & 41 & 53 & 8 & 1 & 30 & 21 & 119 & 199 \\
\hline$\left[\begin{array}{lll}0 & 0.3\end{array}\right] \mathrm{s}$ & 47 & 41 & 7 & 2 & 36 & 26 & 129 & 195 \\
\hline$\left[\begin{array}{lll}0 & 0.4\end{array}\right] \mathrm{s}$ & 44 & 58 & 5 & 2 & 45 & 31 & 114 & 192 \\
\hline$\left[\begin{array}{lll}0 & 0.5\end{array}\right] \mathrm{s}$ & 39 & 46 & 7 & 3 & 45 & 35 & 140 & 192 \\
\hline$\left[\begin{array}{lll}0 & 0.6\end{array}\right] \mathrm{s}$ & 34 & 31 & 8 & 4 & 49 & 40 & 143 & 192 \\
\hline$\left[\begin{array}{lll}0 & 0.7\end{array}\right] \mathrm{s}$ & 31 & 32 & 9 & 4 & 51 & 43 & 157 & 199 \\
\hline$\left[\begin{array}{lll}0 & 0.8\end{array}\right] \mathrm{s}$ & 31 & 24 & 11 & 7 & 51 & 46 & 198 & 238 \\
\hline$\left[\begin{array}{lll}0 & 0.9\end{array}\right] \mathrm{s}$ & 28 & 24 & 15 & 6 & 53 & 50 & 212 & 227 \\
\hline$\left[\begin{array}{ll}0 & 1\end{array}\right] \mathrm{s}$ & 25 & 23 & 11 & 6 & 58 & 54 & 202 & 240 \\
\hline
\end{tabular}

Contralateral ncremental difference
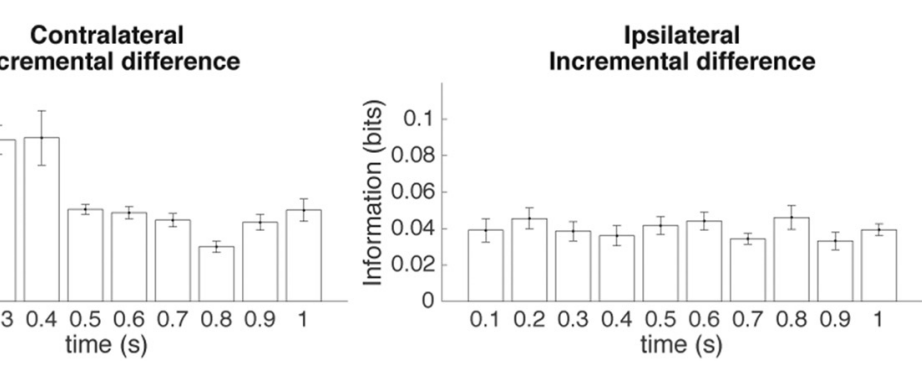

Figure 5. Temporal information content analysis. The MI for each consecutive time window of $0.1 \mathrm{~s}$ duration is shown separately for the CL and IL stimulation patterns.

\section{Limitations of the approach}

The approach we used was previously applied to the analysis of CL inputs in Oddo et al. (2017), but here the decoding of the CL inputs was instead compared with the decoding of a corresponding set of ipsilateral stimulation patterns. Being based on repeatable spatiotemporal patterns of afferent activation, this approach has the advantage that the finest differences in the temporal evolution of the spike responses can be quantified in terms of decoding performance. As discussed previously (Oddo et al., 2017), bypassing the inherent variability inevitably associated with mechanical skin sensor activation (Hayward et al., 2014) allows the study of the central processing of the tactile afferent signals in isolation. A disadvantage is that the input patterns, based on several channels of electrical activation of local primary afferents, to some extent can be argued to be unnatural. To maximize the probability that these patterns were as close to conceivable natural activation as possible, we used a bionic fingertip to generate the specific patterns used, and delivered them using low-intensity electrical skin stimulation (Oddo et al., 2017). These measures, and the approach to mimic the temporal envelope of skin sensor activation occurring under active touch, where, for example, slowly and rapidly adapting receptors have similar activation profiles, makes it possible to argue that the afferent input patterns used fall inside the space of conceivable natural afferent patterns (Oddo et al., 2017). In addition, the very long processing times analyzed (up to $700 \mathrm{~ms}$ after the last stimulation pulse was delivered) makes it likely that most of the effects studied were a reflection of the normal neuronal network propagation for a passively received stimulus. The largest effect of the potentially unnatural levels of synchronicity of tactile afferent activation would be ex- 


\section{A}

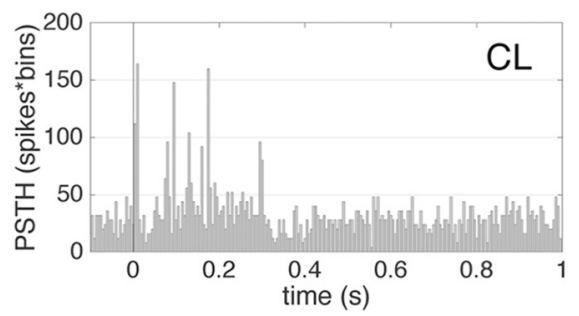

B

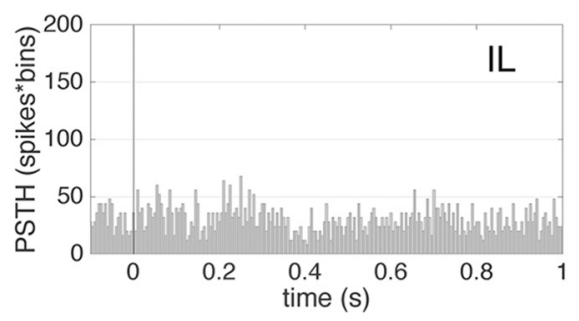

C

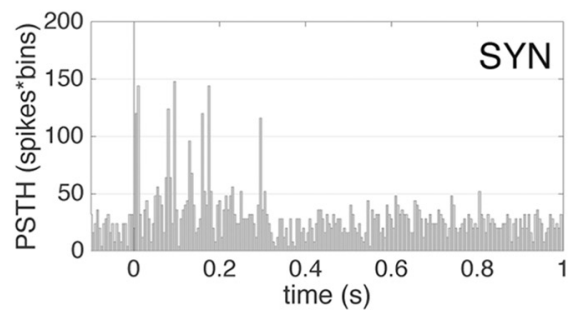

D

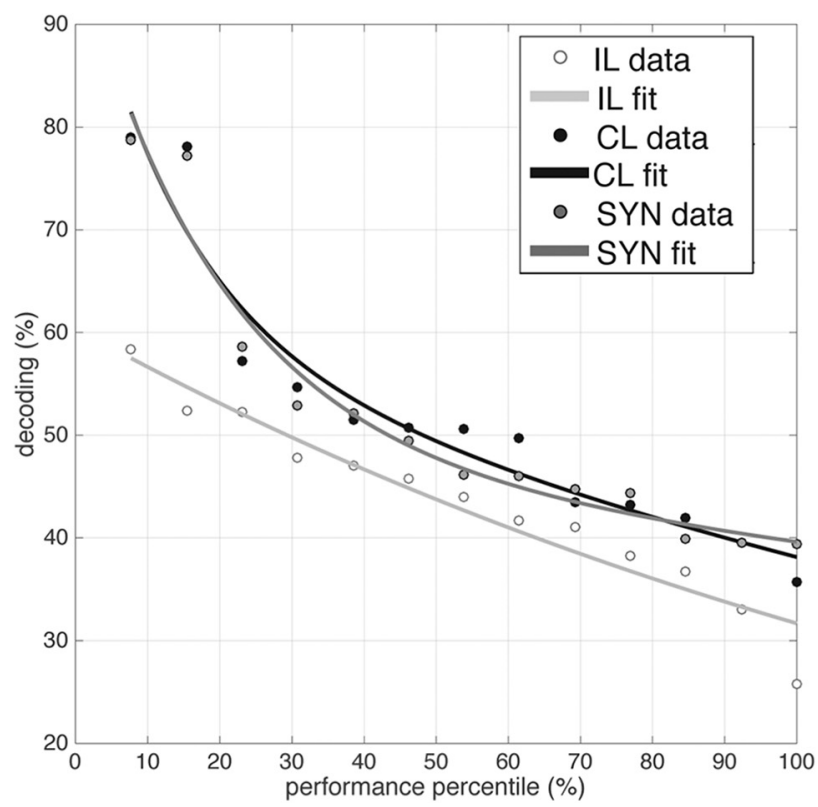

E

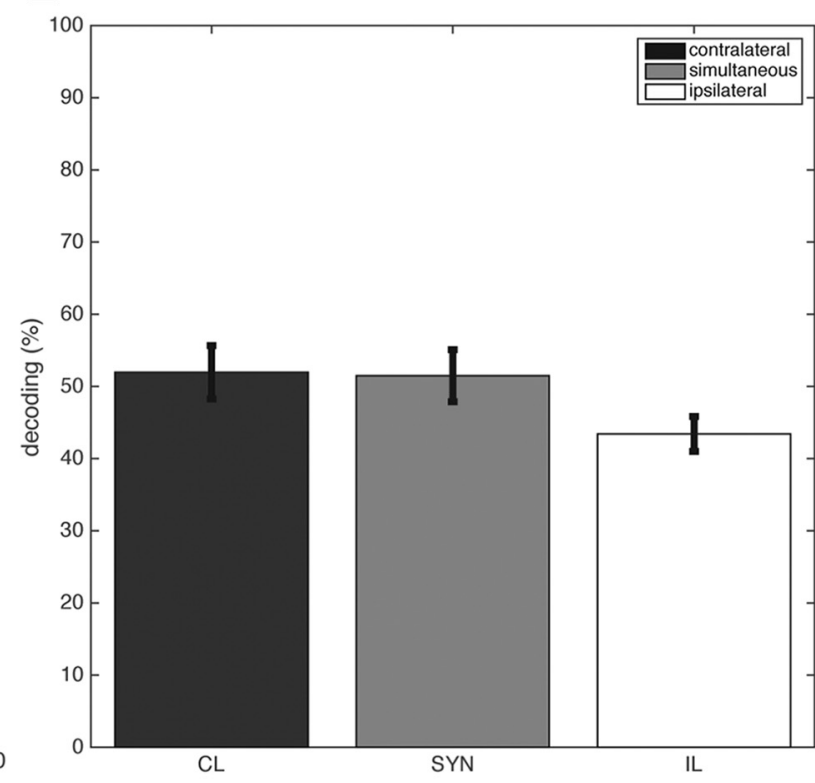

Figure 6. Single neuron decoding analysis during combined bilateral stimulation. $\boldsymbol{A}, \mathrm{PSTH}$ s of the spike responses of a sample neuron to a sample CL stimulation pattern (S5). $\boldsymbol{B}$, PSTH of the same neuron to a sample IL stimulation pattern (S5). C, PSTHs of the two stimulation patterns (CL and IL) delivered in a SYN manner. D, The distribution of the decoding performance and corresponding curve fits for the CL, IL, and SYN stimulation patterns, sorted based on their decoding performance level (expressed as the performance percentile). $\boldsymbol{E}$, The grand averages of the mean decoding for each of the three modes of stimulation. None of the differences were statistically significant $(\mathrm{H}(2)=3, p \gg 0.05$, Kruskal-Wallis test).

pected to be present in the cuneate, less in the thalamus and in the thalamo-cortical activation (i.e., within the first $10 \mathrm{~ms}$ of each pulse).

We explored only a small part of the parametric space of possible spatiotemporal tactile afferent input patterns (i.e., the patterns obtained from eight identical simulated active touch conditions of the fingertip of the second forepaw digit) with probes differing only with respect to their surface curvature. It is of course likely that larger differences in the spatiotemporal input patterns would have resulted in better decoding performance and possibly resulted in larger decoding differences between CL and IL inputs. Nevertheless, the most remarkable finding of the present study is the fact that the IL inputs could be decoded with such high accuracy given the quite small differences between them.

If the anesthesia facilitated or inhibited the network propagation of the evoked activity, and thereby improved or degraded the decoding, remains an open question. It has been shown that the latency of the responses in the SC were similar in anesthetized rats (Shuler et al., 2001) and awake rats (Wiest et al., 2005), even though the bilateral interactions appeared more dynamic in the awake rats (Wiest et al., 2005). The long latency responses, which clearly contributed an important part of the IL decoding (Fig. 4), may be gated out at least under some conditions in the awake state, but then again under other conditions they may instead be gated in. In the whisker system of the awake rat, the latency times for behavioral responses in a discrimination task for bilateral stimulation frequencies were found to be well $<0.5 \mathrm{~s}$ (Mayrhofer et al., 2013), which would seem hard to support with the decoding times reported here. However, a difference with the present study is that instead of discriminating one frequency from another, here the requirement for the neurons was to be able to tell which one of the eight stimulation patterns was presented to the IL side. Moreover, some information about the type of IL input in the neuron existed already after $100 \mathrm{~ms}$, even though the decoding performance increased substantially with integration time.

\section{Potential pathways involved}

The CL response amplitudes were significantly higher than those of the IL stimulations, and the response latency times were much shorter (Fig. 2C,D). Weaker and slower ipsilateral responses were observed in previous studies using both whisker and forepaw/ hindpaw stimulations, which have been taken as an indication of a corticocortical transmission between hemispheres as the primary route for IL inputs (Armstrong-James and George, 1988; Tutunculer et al., 2006; Moxon et al., 2008). Our results do not add information to this issue. However, in our study the observed highly variable latency times for the CL input (CL minimum, $0.005 \mathrm{~s}$; CL maximum, $0.44 \mathrm{~s}$ ), which were expected because of the low stimulation intensities used, suggested that the CL responses in the neocortical neurons did not primarily rely on di- 
rect thalamocortical afferents but may to a larger extent be mediated by corticocortical routes (Fig. 2D).

\section{Behaviorally observed phenomena of bilateral haptic integration}

In humans, tactile stimulation patterns received passively to one digit generate activity that spreads bilaterally in the neocortex, with a focus on the SC (Genna et al., 2017), and the same applies to the rodent whisker systems (Ferezou et al., 2007). A recent study (Dupin et al., 2015) indicates that what we perceive as the sensory consequences of moving one hand can be influenced by passive tactile stimulation of the other, provided that the kinesthetic and tactile events coincide in time. This suggests that bilateral tactile integration may occur early in the neocortical processing (Mayrhofer et al., 2013; Tamè et al., 2015, 2016). For the rat whisker system, bilateral activation and bilateral sensory feedback may be more of a rule in everyday life (Saraf-Sinik et al., 2015) than for distal digit skin on the digits. This is interesting in relation to the differences in response latencies and amplitudes with the CL and IL inputs that we observed compared with the whisker system, where such differences have been reported to be substantially smaller (Wiest et al., 2005). Unless the differences have a pure methodological explanation, they could depend on stronger interhemispheric linkages that could arise because of a more consistent occurrence of bilateral activation for the whiskers than for the digits.

\section{Possible significance of differences in decoding times: ambient versus local information}

The mean CL and IL decoding increased approximately linearly with time as measured up to $1.0 \mathrm{~s}$ (i.e., up to $0.7 \mathrm{~s}$ after the skin stimulation terminated; Fig. 4C; Oddo et al., 2017). This result indicates that an induction of long-lasting aftereffects in the sensory processing circuitry may be triggered by the external input. Such aftereffects seem to reflect at least in part the cortical network pathways of interneuronal information transfer used in awake processing, which can be illustrated by the relatively fixed neuronal recruitment order in evoked responses as well as in spontaneous up-states (Luczak et al., 2009) and which are not altered under anesthesia (Luczak and Barthó, 2012). Interestingly, the information content for the CL input was higher for the time window $0.1-0.4 \mathrm{~s}$, when the peripheral stimulation was active, but this was not true for the IL input (Fig. 5). Hence, whereas the average neuronal decoding performance eventually became essentially equivalent for CL and IL inputs, the CL input reached higher decoding levels at a much shorter processing time than the IL input. This indicates that the processing of the CL input is more effective than that for the IL input, a difference that is likely due to the higher responsiveness of the local circuitry to the direct peripheral input from the CL side. Also the experiments with coactivation of CL and IL inputs (Fig. 6) suggested that the CL decoding is dominating the processing in the local neocortical neuronal network. Our results show that the local circuitry contains highly specific information about the quality of the tactile information on the IL side, information that may, for example, be important to create predictions or disambiguation for input from the CL side (Dupin et al., 2015). To what extent that information is used in the awake state may be gated by context-dependent mechanisms in the neocortical circuitry and is an important issue for future research to clarify.

\section{References}

Armstrong-James M, George MJ (1988) Bilateral receptive fields of cells in rat Sml cortex. Exp Brain Res 70:155-165. Medline
Brasil-Neto JP, de Lima AC (2008) Sensory deficits in the unaffected hand of hemiparetic stroke patients. Cogn Behav Neurol 21:202-205. CrossRef Medline

Dupin L, Hayward V, Wexler M (2015) Direct coupling of haptic signals between hands. Proc Natl Acad Sci U S A 112:619-624. CrossRef Medline

Ferezou I, Haiss F, Gentet LJ, Aronoff R, Weber B, Petersen CC (2007) Spatiotemporal dynamics of cortical sensorimotor integration in behaving mice. Neuron 56:907-923. CrossRef Medline

Genna C, Oddo CM, Fanciullacci C, Chisari C, Jörntell H, Artoni F, Micera S (2017) Spatiotemporal dynamics of the cortical responses induced by a prolonged tactile stimulation of the human fingertips. Brain Topogr 30: 473-485. CrossRef Medline

Geyer S, Schleicher A, Zilles K (1999) Areas 3a, 3b, and 1 of human primary somatosensory cortex: 1 . Microstructural organization and interindividual variability. Neuroimage 10:63-83. CrossRef Medline

Hayward V, Terekhov AV, Wong SC, Geborek P, Bengtsson F, Jörntell H (2014) Spatio-temporal skin strain distributions evoke low variability spike responses in cuneate neurons. J R Soc Interface 11:20131015. CrossRef Medline

Ince RA, Mazzoni A, Petersen RS, Panzeri S (2010) Open source tools for the information theoretic analysis of neural data. Front Neurosci 4:62-70. CrossRef Medline

Iwamura Y (2000) Bilateral receptive field neurons and callosal connections in the somatosensory cortex. Philos Trans R Soc Lond B Biol Sci 355:267273. CrossRef Medline

Iwamura Y, Iriki A, Tanaka M (1994) Bilateral hand representation in the postcentral somatosensory cortex. Nature 369:554-556. CrossRef Medline

Iwamura Y, Tanaka M, Iriki A, Taoka M, Toda T (2002) Processing of tactile and kinesthetic signals from bilateral sides of the body in the postcentral gyrus of awake monkeys. Behav Brain Res 135:185-190. CrossRef Medline

Luczak A, Barthó P (2012) Consistent sequential activity across diverse forms of UP states under ketamine anesthesia. Eur J Neurosci 36:2830 2838. CrossRef Medline

Luczak A, Barthó P, Harris KD (2009) Spontaneous events outline the realm of possible sensory responses in neocortical populations. Neuron 62:413425. CrossRef Medline

Magri C, Whittingstall K, Singh V, Logothetis NK, Panzeri S (2009) A toolbox for the fast information analysis of multiple-site LFP, EEG and spike train recordings. BMC Neurosci 10:81. CrossRef Medline

Mayrhofer JM, Skreb V, von der Behrens W, Musall S, Weber B, Haiss F (2013) Novel two-alternative forced choice paradigm for bilateral vibrotactile whisker frequency discrimination in head-fixed mice and rats. J Neurophysiol 109:273-284. CrossRef Medline

Moxon KA, Hale LL, Aguilar J, Foffani G (2008) Responses of infragranular neurons in the rat primary somatosensory cortex to forepaw and hindpaw tactile stimuli. Neuroscience 156:1083-1092. CrossRef Medline

Nieuwenhuys R, Voogd J, Van Huijzen C (2008) The human central nervous system: a synopsis and atlas. Berlin, Germany: Springer.

Oddo CM, Mazzoni A, Spanne A, Enander JM, Mogensen H, Bengtsson F, Camboni D, Micera S, Jörntell H (2017) Artificial spatiotemporal touch inputs reveal complementary decoding in neocortical neurons. Sci Rep 8:45898. CrossRef Medline

Panzeri S, Brunel N, Logothetis NK, Kayser C (2010) Sensory neural codes using multiplexed temporal scales. Trends Neurosci 33:111-120. CrossRef Medline

Pidoux B, Verley R (1979) Projections on the cortical somatic I barrel subfield from ipsilateral vibrissae in adult rodents. Electroencephalogr Clin Neurophysiol 46:715-726. CrossRef Medline

Quian Quiroga R, Panzeri S (2009) Extracting information from neuronal populations: information theory and decoding approaches. Nat Rev Neurosci 10:173-185. CrossRef Medline

Ruben J, Schwiemann J, Deuchert M, Meyer R, Krause T, Curio G, Villringer K, Kurth R, Villringer A (2001) Somatotopic organization of human secondary somatosensory cortex. Cereb Cortex 11:463-473. CrossRef Medline

Saraf-Sinik I, Assa E, Ahissar E (2015) Motion makes sense: an adaptive motor-sensory strategy underlies the perception of object location in rats. J Neurosci 35:8777-8789. CrossRef Medline

Schnitzler A, Salmelin R, Salenius S, Jousmäki V, Hari R (1995) Tactile information from the human hand reaches the ipsilateral primary somatosensory cortex. Neurosci Lett 200:25-28. CrossRef Medline

Schomer DL, Da Silva FL (2012) Niedermeyer's electroencephalography: 
basic principles, clinical applications, and related fields. Philadelphia, PA: Lippincott Williams \& Wilkins.

Shimazaki H, Shinomoto S (2010) Kernel bandwidth optimization in spike rate estimation. J Comput Neurosci 29:171-182. CrossRef Medline

Shuler MG, Krupa DJ, Nicolelis MA (2001) Bilateral integration of whisker information in the primary somatosensory cortex of rats. J Neurosci 21: 5251-5261. Medline

Shuler MG, Krupa DJ, Nicolelis MA (2002) Integration of bilateral whisker stimuli in rats: role of the whisker barrel cortices. Cereb Cortex 12:86-97. CrossRef Medline

Tamè L, Pavani F, Papadelis C, Farnè A, Braun C (2015) Early integration of bilateral touch in the primary somatosensory cortex. Hum Brain Mapp 36:1506-1523. CrossRef Medline
Tamè L, Braun C, Holmes NP, Farnè A, Pavani F (2016) Bilateral representations of touch in the primary somatosensory cortex. Cogn Neuropsychol 33:48-66. CrossRef Medline

Tutunculer B, Foffani G, Himes BT, Moxon KA (2006) Structure of the excitatory receptive fields of infragranular forelimb neurons in the rat primary somatosensory cortex responding to touch. Cereb Cortex 16 : 791-810. CrossRef Medline

Wiest MC, Bentley N, Nicolelis MA (2005) Heterogeneous integration of bilateral whisker signals by neurons in primary somatosensory cortex of awake rats. J Neurophysiol 93:2966-2973. CrossRef Medline

Zhu Z, Disbrow EA, Zumer JM, McGonigle DJ, Nagarajan SS (2007) Spatiotemporal integration of tactile information in human somatosensory cortex. BMC Neurosci 8:21. CrossRef Medline 